\title{
EFFECT OF TWO-DIMENSIONAL ELECTRON GAS ON THE D.C. CHARACTERISTICS OF InAlAs/InGaAs DOUBLE HETEROJUNCTION BIPOLAR TRANSISTORS
}

\author{
ChaO-Hsing Huang and HaO-Hsiung Lin \\ Department of Electrical Engineering, National Taiwan University, Taipei, Taiwan, R. O. China
}

(Received 7 February 1993)

\begin{abstract}
In this study, the characteristics of InAlAs/InGaAs double heterojunction bipolar transistors (DHBT's) with abrupt and graded base-collector junction structures are investigated. We find that because of the large conduction band discontinuity between the InGaAs base and the InAlAs collector, the base-collector abrupt heterojunction notch will accumulate a two-dimensional electron gas (2DEG) and the heterojunction spike will be pushed up. Therefore, poor knee-shape and reach-through effects appear in the device even with a $500 \AA$-thick undoped InGaAs layer sandwiched between the base and collector. This finding has never been observed in DHBT's with small band discontinuity material systems such as InP/InGaAs and AlGaAs/GaAs. The InAlAs/InGaAs DHBT with a graded base-collector junction shows no knee-shape and reach-through effects, because the heterojunction notch has been smoothed out.
\end{abstract}

\section{INTRODUCTION}

Recently, heterojunction bipolar transistors (HBT's) of InP-related materials have received a great deal of interest. Their base materials, i.e. InGaAs and In GaAsP[1-4], have superior electrical properties to GaAs. And the material system is suitable for the long-wavelength optical communications[5]. So far, high current gain and high unit current gain frequency have been demonstrated[6-8]. Among the above alloys, the InAlAs/InGaAs heterojunction system has the largest conduction-band discontinuity[9] which can be used for a hot-electron launching pad[10,11]. However, InAlAs/InGaAs HBT's suffer from the problem of poor breakdown characteristics. The main breakdown mechanism is the hot-electronenhanced impact ionization taking place in the narrow band gap InGaAs base-collector junction[12]. In order to solve this problem, large band gap InAlAs collector has been adopted in this study. This method can preserve the injected hot electrons and improve the breakdown characteristics simultaneously.

Because the conduction band discontinuity of the InAlAs/InGaAs system is as large as $0.53 \mathrm{eV}$, care must be taken in the design of the base-collector heterojunction structure in order to avoid the kneeshape and reach-through effects reported previously[13]. In this study, two layer structures are investigated. The first one is to insert an undoped low-band-gap spacer layer between the low-band-gap base and the high-band-gap collector. The main principle of this simple method is to utilize the voltage drop across the uncioped spacer layer so as to suppress the heterojunction spike[14]. It has been successfully demonstrated in the $\mathrm{AlGaAs} / \mathrm{GaAs}$ DHBT[13] and in the InP/InGaAs DHBT[15]. It is interesting that the InAlAs/InGaAs DHBT, with much larger conduction band discontinuity than that of the above two material systems, shows different characteristics. Our study indicates that this method does not work effectively in InAIAs/InGaAs DHBT, and that there is another mechanism taking place in this special system of large conduction band discontinuity. All the results show that a two-dimensional electron gas (2DEG) will accumulate in the base-collector heterojunction notch and thus push up the heterojunction spike. Two different spacer-layer thicknesses, $300 \AA$ and $500 \AA$, were used. Both of them show poor knee-shape and reach-through effects. Further increase in spacer layer thickness is limited by the hot-electron-enhanced impact ionization taking place in the low-band-gap spacer layer.

The second structure utilizes junction grading. It is found that because the heterojunction spike and notch have been smoothed-out, the output characteristics of DHBT are significantly improved and show no knee-shape and reach-through effects.

\section{EXPERIMENT}

The epilayers of the HBT's were grown using a VG V80-H Mark-II solid-source molecular beam epitaxy (MBE) system. The starting material was Dowa mining (100) Sn-doped $n^{+}-\operatorname{InP}$ substrate. The details of the DHBT structures are summarized in Table 1. Four epiwafers were grown for DHBT device fabrication. Among them, R423, R424, and R425 were grown in the same batch and on the same day. R221 was grown previously but with the same structure as R423. The differences between the structures are on their base collector junctions. For devices R423 and R424, undoped InGaAs spacer layers of 300 and 
Table 1. The layer structure of InAlAs/InGaAs DHBT

\begin{tabular}{|c|c|c|c|c|c|c|c|}
\hline \multirow[b]{2}{*}{ Layer } & \multirow[b]{2}{*}{ Material } & \multirow[b]{2}{*}{ Dopant } & \multicolumn{4}{|c|}{ Thickness $(\mu \mathrm{m})$} & \multirow[b]{2}{*}{ Doping $\left(\mathrm{cm}^{-3}\right)$} \\
\hline & & & R221 & R423 & R424 & R425 & \\
\hline Cap 1 & InAs & $\mathrm{Si}$ & \multicolumn{2}{|c|}{0.015} & 0.015 & 0.015 & $3 \times 10^{19}$ \\
\hline Cap 3 & InAlAs & $\mathbf{S i}$ & \multicolumn{2}{|c|}{0.05} & 0.05 & 0.05 & $3 \times 10^{19}$ \\
\hline Emitter & InAlAs & Si & \multicolumn{2}{|c|}{0.15} & 0.15 & 0.15 & $1 \times 10^{17}$ \\
\hline Spacer & InGaAs & Undoped & \multicolumn{2}{|c|}{0.03} & 0.03 & 0.03 & $\sim 10^{15}(n)$ \\
\hline Base & InGaAs & $\mathrm{Be}$ & \multicolumn{2}{|c|}{0.15} & 0.15 & 0.15 & $4 \times 10^{18}$ \\
\hline Graded layer & InGaAlAs & $\mathrm{Si}$ & \multicolumn{2}{|c|}{-} & - & 0.05 & $\sim 10^{15}(n)$ \\
\hline Collector & InAlAs & $\mathrm{Si}$ & \multicolumn{2}{|c|}{0.4} & 0.4 & 0.4 & $1 \times 10^{17}$ \\
\hline Buffer & InAlAs & $\mathrm{Si}$ & \multicolumn{2}{|c|}{0.2} & 0.2 & 0.2 & $3 \times 10^{19}$ \\
\hline Substrate & $\ln \mathrm{P}$ & $\mathrm{Sn}$ & \multicolumn{2}{|c|}{350} & 350 & 350 & $1 \times 10^{18}$ \\
\hline
\end{tabular}

$500 \AA$-thick were inserted into their base-collector junction, respectively. For device R425, besides the $300 \AA$-thick undoped InGaAs spacer layer, a $500 \AA$ thick band-gap graded layer was grown between the collector and the undoped InGaAs spacer layer. A pulsed molecular beam method[16] was used to prepare the graded layer. The emitter-base junction structures of all three devices consist of abrupt In AlAs/InGaAs heterojunctions with a $300 \AA$-thick undoped spacer layer. This design preserves the heterojunction spike for hot electron injection. Besides the above devices, a single heterojunction bipolar device (SHBT), i.e. device R291, is also involved in this study. Its layer structure is summarized in Table 2. The growth temperature for these devices was $500^{\circ} \mathrm{C}$.

To fabricate the HBT's, an $1500 \AA$-thick $\mathrm{Au}-\mathrm{Ge}-\mathrm{Ni}$ was firstly deposited and lifted-off from the cap layer for the emitter ohmic contact. A 1 $\mathrm{H}_{3} \mathrm{PO}_{4}: 1 \quad \mathrm{H}_{2} \mathrm{O}_{2}: 20 \quad \mathrm{H}_{2} \mathrm{O}$ solution was then used to remove both the cap and the emitter layer to expose the base layer. This was followed by a $1200 \AA$-thick $\mathrm{Au} / \mathrm{Ti}$ nonalloyed contact deposition and lifted-off from the base layer. Finally, the devices were isolated by mesa etching in a $1 \quad \mathrm{H}_{3} \mathrm{PO}_{4}: 1 \quad \mathrm{H}_{2} \mathrm{O}_{2}: 20 \quad \mathrm{H}_{2} \mathrm{O}$ solution. The area of the emitter-base junction and base-collector junction are $3.6 \times 10^{-5}$ and $1.8 \times 10^{-4} \mathrm{~cm}^{2}$, respectively.

\section{RESULTS AND DISCUSSIONS}

In the design of DHBT with abrupt base-collector junction, the thickness of the undoped InGaAs spacer layer was considered as an important parameter. Because the voltage drop across the layer has to be large enough to suppress the heterojunction spike, two different thicknesses were chosen and their effects were compared experimentally. Before the experiment, theoretical calculation based on the depletion approximation and previous experimental studies on AlGaAs/GaAs DHBT[13] showed that these spacer layers can provide enough band bending to eliminate knee-shape and reach-through effects resulting from the base-collector heterojunction spike.

Figure 1(a) and (b) show the common emitter output characteristics of R423 and R424, respectively. From the plots, their current gains are 480 and
300, respectively. As can be seen, the breakdown voltages of R423 and R424 are about 5 and $3 \mathrm{~V}$, respectively. These breakdown voltages are much larger than those for InAlAs/InGaAs HBTs with InGaAs collector[7]. From the inserts of Fig. 1, which show the detailed $I-V$ characteristics around the zero point, it is clear that knee-shape and reach-through effects do exist in these devices, which is in contradiction to our calculation. This is because the heterojunction spike of the base-collector junction is not successfully suppressed by spacer layer band bending. Because of the large conduction band discontinuity, the $p^{+}$-InGaAs/ $u$-InGaAs/ $N$-InAlAs base-collector heterojunction must have a two-dimensional electron gas (2DEG) stored in its notch. This 2DEG was not considered in our previous calculation and should be responsible for these unexpected effects. Its effects are schematically illustrated in Fig. $2(a, b)$. Figure 2(a) shows the base-collector heterojunction band diagram without the $2 \mathrm{DEG}$, i.e. the result of our theoretical calculation based on the depletion approximation. As can be seen, the voltage drop across the undoped InGaAs spacer layer is larger than the conduction band discontinuity of the heterojunction, and the hidden potential spike does not influence the collector currents as demonstrated in AlGaAs/GaAs DHBT[13] and InP/InGaAs DHBT[15]. In Fig. 2(b), 2DEG charges have been included in the base-collector heterojunction notch. The source of these electrons is emitter-base injection. When the electrons pass the base-collector junction, some of them are trapped in the heterojunction notch. More importantly, the trapped charges are negative and can replace part of the impurity charges in the $\boldsymbol{P}^{+}-\mathrm{InGaAs}$ depletion region. This will

Table 2. The layer structure of InAlAs/InGaAs SHBT R291

\begin{tabular}{llccc}
\hline Layer & Material & Dopant & Thickness $(\mu \mathrm{m})$ & $\begin{array}{c}\text { Doping } \\
\left(\mathrm{cm}^{-3}\right)\end{array}$ \\
\hline Cap 1 & InAs & $\mathrm{Si}$ & 0.015 & $3 \times 10^{19}$ \\
Cap 2 & InGaAs & $\mathrm{Si}$ & 0.05 & $3 \times 10^{19}$ \\
Cap 3 & InAlAs & $\mathrm{Si}$ & 0.1 & $3 \times 10^{19}$ \\
Emitter & InAlAs & $\mathrm{Si}$ & 0.15 & $1 \times 10^{17}$ \\
Spacer & InGaAs & Undoped & 0.05 & $\sim 10^{15}(n)$ \\
Base & InGaAs & $\mathrm{Be}$ & 0.15 & $2 \times 10^{18}$ \\
Collector & InGaAs & Undoped & 0.5 & $\sim 10^{15}(n)$ \\
Hi-Collector & InGaAs & $\mathrm{Si}$ & 0.25 & $1 \times 10^{17}$ \\
Buffer & InGaAs & $\mathrm{Si}$ & 0.25 & $3 \times 10^{19}$ \\
Substrate & InP & $\mathrm{Sn}$ & 350 & $1 \times 10^{18}$ \\
\hline
\end{tabular}


(a)
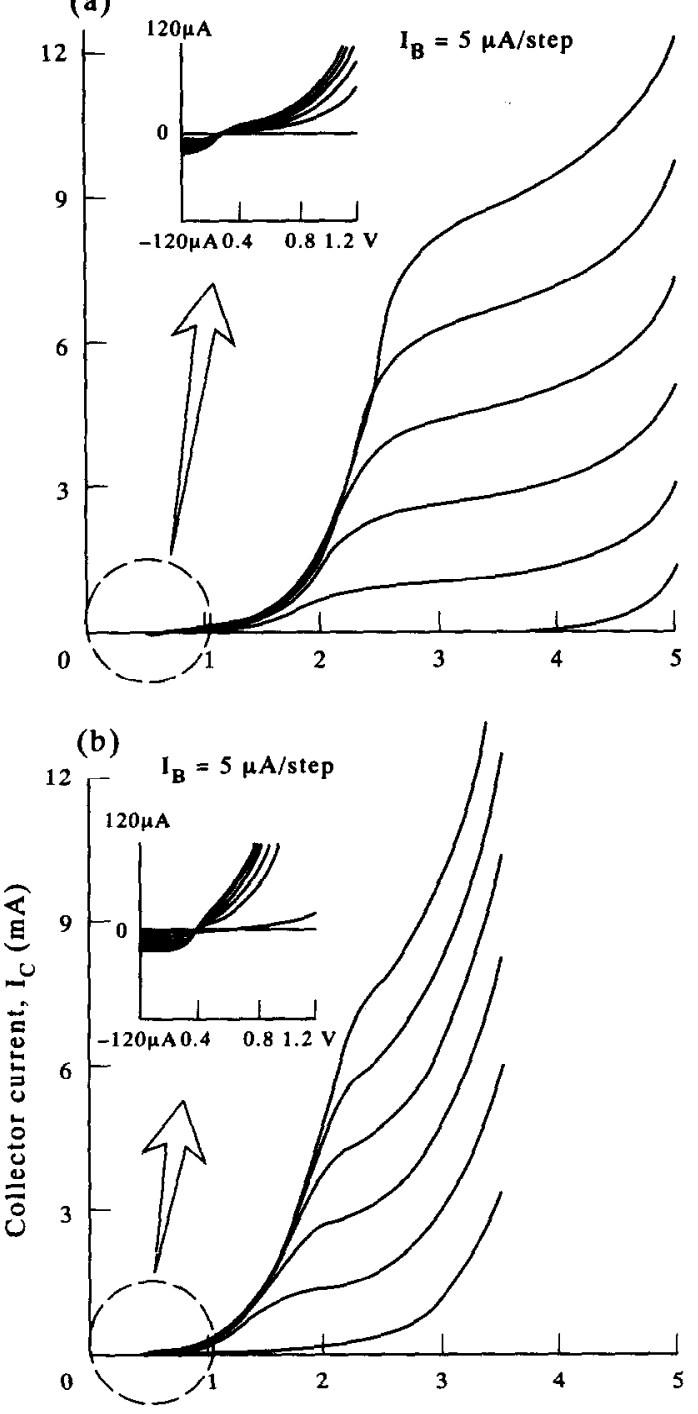

(c) $120 \mu \mathrm{A}$

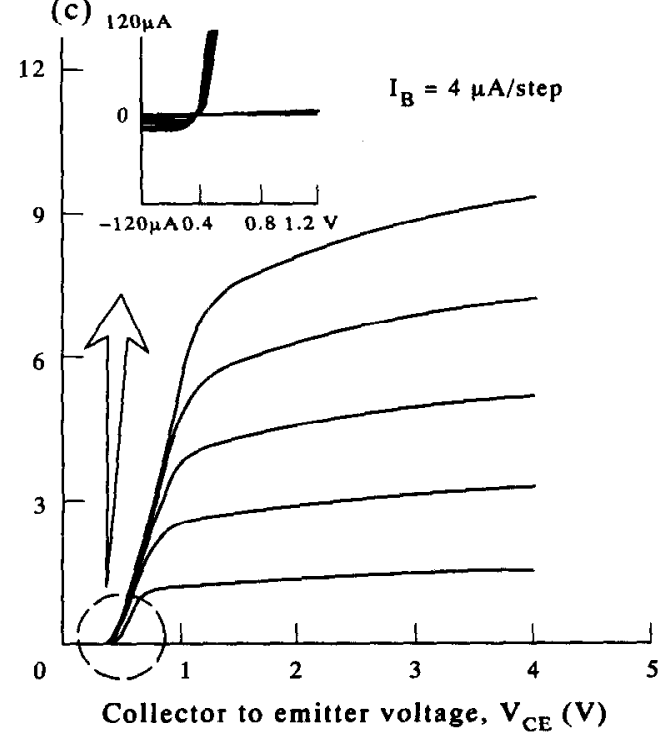

Fig. 1 (a)

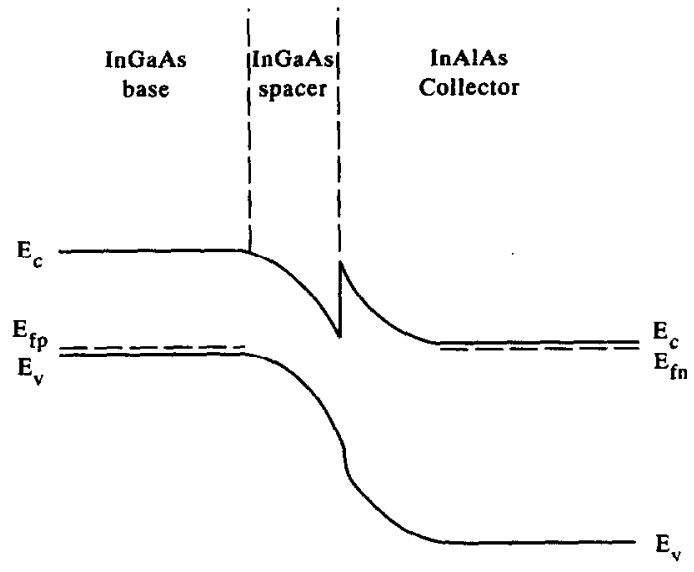

(b)

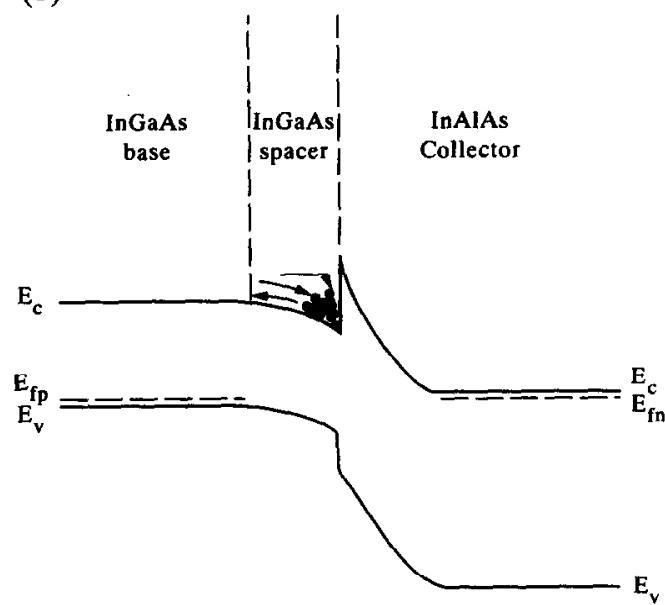

Fig. 2. (a) The base-collector heterojunction band diagram of InAlAs/InGaAs DHBT in which 2DEG is not considered and the heterojunction spike is suppressed. (b) The band diagram with the consideration of 2DEG and a pushed-up heterojunction spike which results in knee-shape and reachthrough effects.

result in a decrease in the voltage drop across the InGaAs spacer layer and the $P^{+}-$InGaAs depletion region. Since the bias voltage of the base-collector junction remains unchanged, the voltage drop across the $N$-InAlAs depletion region must increase. The heterojunction spike is thus pushed up and begins to reflect electrons back to the base. This will cause a decrease in the transport factor of the transistor and an increase in the minority carrier concentration of

Fig. 1. The common emitter output characteristics of DHBT's, (a) R423 with 300 \& -thick spacer layer, (b) R424 with $500 \AA$-thick spacer layer, and (c) R425 with 300 A-thick spacer layer and $500 \AA$-thick graded layer inserted between the base-collector junctions. The inserts of the figures show the detailed $I-V$ characteristics around zero point. In the inserts of (a) and (b), knee-shape and reach-through effects can be clearly seen, while in the insert of (c), collector current rises sharply. 
(a)

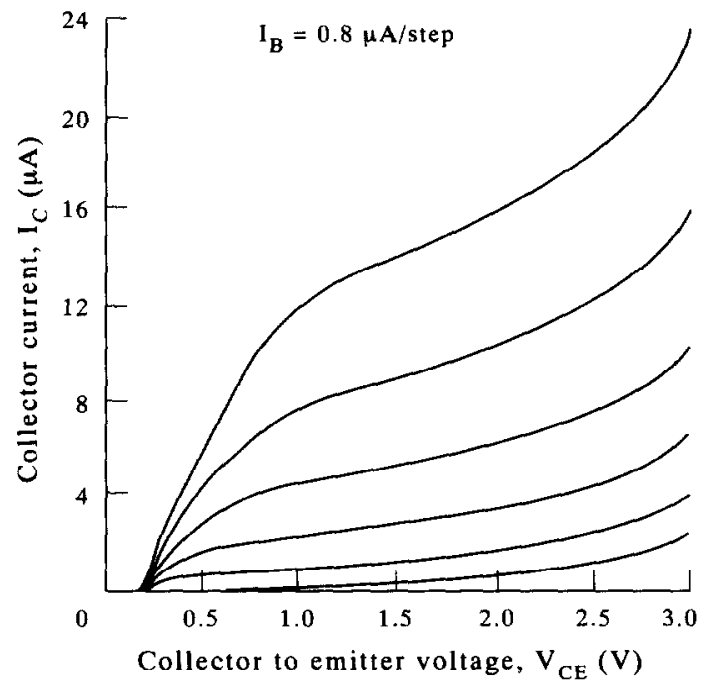

(b)

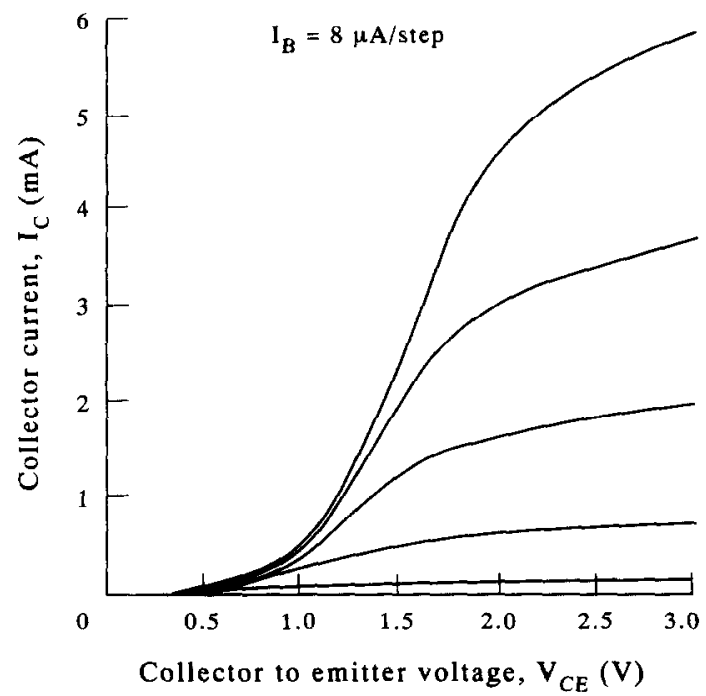

Fig. 3. The common emitter output characteristics of DHBT R221 in (a) low current region, and (b) in high current region. The base-collector junction structure of $R 221$ is abrupt and with $300 \AA$-thick undoped spacer layer.

the base region. The sheet density of the 2DEG must also increase and the heterojunction spike is thus further pushed up. However, the lowered voltage drop across the undoped InGaAs spacer layer will allow more electrons to escape from the notch. This, in turn, will pull down the heterojunction spike. Finally, these two mechanisms may reach a balance; a higher heterojunction spike energy is shown in Fig. 2(b).

From Fig. 1, we also noted that R423 has worse knee-shape and reach-through effects than R424. This is because that the $500 \AA$-thick spacer layer of R424 has more band bending than that of the $300 \AA$-thick spacer layer, and hence the heterojunction spike energy of R424 is lower than that of R423.
Further increase of the spacer thickness may improve the reach-through effect of the output characteristics. However, the smaller breakdown voltage of R424 reveals that the $500 \AA$-thick spacer layer has been thick enough to permit considerable hot-electronenhanced impact ionization. Under this consideration, we turned to the graded junction structure. Figure 1(c) shows the common emitter output characteristics of device R425. It is clearly seen that the knee-shape effect and reach-through effect indeed disappear. In the saturation region, the collector current rises very sharply. This indicates that the heterojunction spike is smoothed out. The breakdown voltage of $R 425$ is over $5 \mathrm{~V}$ which is good enough for device application. Its current gain and base doping concentration are 520 and $4 \times 10^{18} / \mathrm{cm}^{3}$, respectively.

From the discussion of Fig. 2, the sheet density of the 2DEG stored in the heterojunction notch is determined by two factors, the conduction band discontinuity of the heterojunction and the collector current density. Notice that the very large conduction band discontinuity of the InAlAs/InGaAs system will cause a deep heterojunction notch and thus a low 2DEG escape rate and a high heterojunction spike and thus a high 2DEG trapping rate as shown in Fig. 2(b). This makes a high density 2DEG accumulate in the heterojunction notch and renders the undoped InGaAs spacer layer useless. The same mechanism may take place in the $\mathrm{AlGaAs} / \mathrm{GaAs}$ and the InP/InGaAs system. However the much smaller conduction band discontinuity does not make the 2DEG density large enough to cause problems.

The second factor to influence the 2DEG density and thus the heterojunction spike energy is the collector current. The larger the collector current, the higher the 2DEG density and the spike energy. It

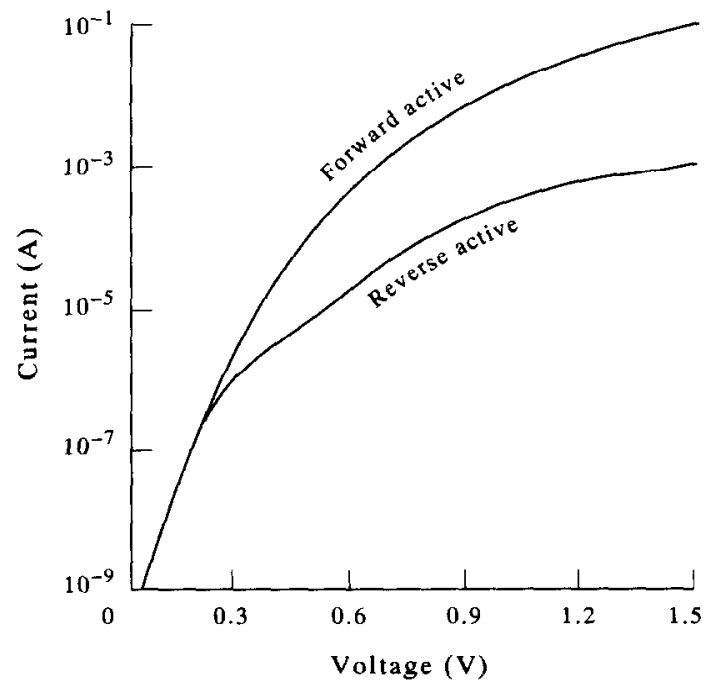

Fig. 4. The Gummel plot of InAlAs/InGaAs single heterojunction bipolar transistor R291. In this device, there is a $500 \AA$-thick undoped InGaAs layer inserted between the emitter and base junction. 
follows that in low current region, the reach-through effect should become less significant because stored 2DEG is less and the heterojunction spike energy is lower. Figure 3(a) and (b) show the low current and high current output characteristics of DHBT R221 whose base-collector junction structure is abrupt and with $300 \AA$-thick undoped spacer layer. Clearly, the results support this point. The other evidence for a current-dependent 2DEG is shown in Fig. 4, which is the Gummel plot of an InAlAs/InGaAs single heterojunction bipolar transistor R291. In this device, there is a $500 \AA$-thick undoped InGaAs layer inserted between the emitter and base junction. As can be seen, the collector current of the forward active mode has a close to unity junction ideality factor in the low current region. The saturation current density is $1.86 \times 10^{-6} \mathrm{~A} / \mathrm{cm}^{2}$, which is consistent with the value of InGaAs $p^{+} / n$ homojunction bipolar transistor fabricated in our laboratory. In addition, the emitter current of the reverse active mode coincides with the collector current in low current region. This is the reciprocity of bipolar transistors.

These behaviors suggest that in the low current region, there is no heterojunction spike existing in the InAlAs/InGaAs heterojunction. However, when the current increases above $5 \times 10^{-7} \mathrm{~A}$, the curve of the reverse active mode deviates from the slope for a unity junction ideality factor. Notice that in the reverse active mode, the injection current is from the InGaAs base-collector homojunction and the In AlAs/InGaAs emitter-base junction is kept at zerobiased. Although the bias of the emitter-base heterojunction is fixed, the increase of injection current induces the 2DEG density and thus pushes up the emitter-base heterojunction spike. The rising spike provides extra barrier height for the injection current and reduces its slope as shown in Fig. 4. The result also indicates that $500 \AA$-thick spacer layer can not remove the reach-through effect and is consistent with the result of DHBT R424.

In order to further study the effect of the 2DEG and its current dependent behavior, the common base output characteristics of DHBT's R423, R424, and R425 were measured and are shown in Fig. 5(a), (b) and (c), respectively. Again, the curves of R423 and R424 show similar knee-shape and reach-through behaviors, while the behavior of device R425 is absolutely the same as that of conventional bipolar device. From these plots, we can find the base-collector voltage at which the collection current reaches its saturation value, i.e. the value at its active region.

For convenience, this voltage is called the suppression voltage, because the heterojunction spike is suppressed and the transistor reaches its active region. Clearly, this suppression voltage is an evaluation of how serious the reach-through effect is. The suppression voltages of all three devices are plotted as functions of emitter current in Fig. 6. As can be seen, the suppression voltages of R423 and R424 are

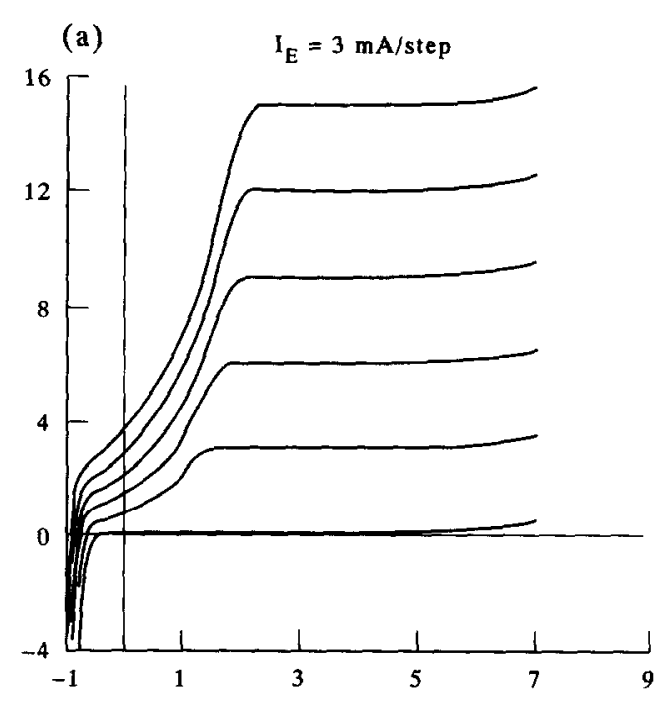

(b) $\quad \mathrm{I}_{\mathrm{E}}=3 \mathrm{~mA} / \mathrm{step}$
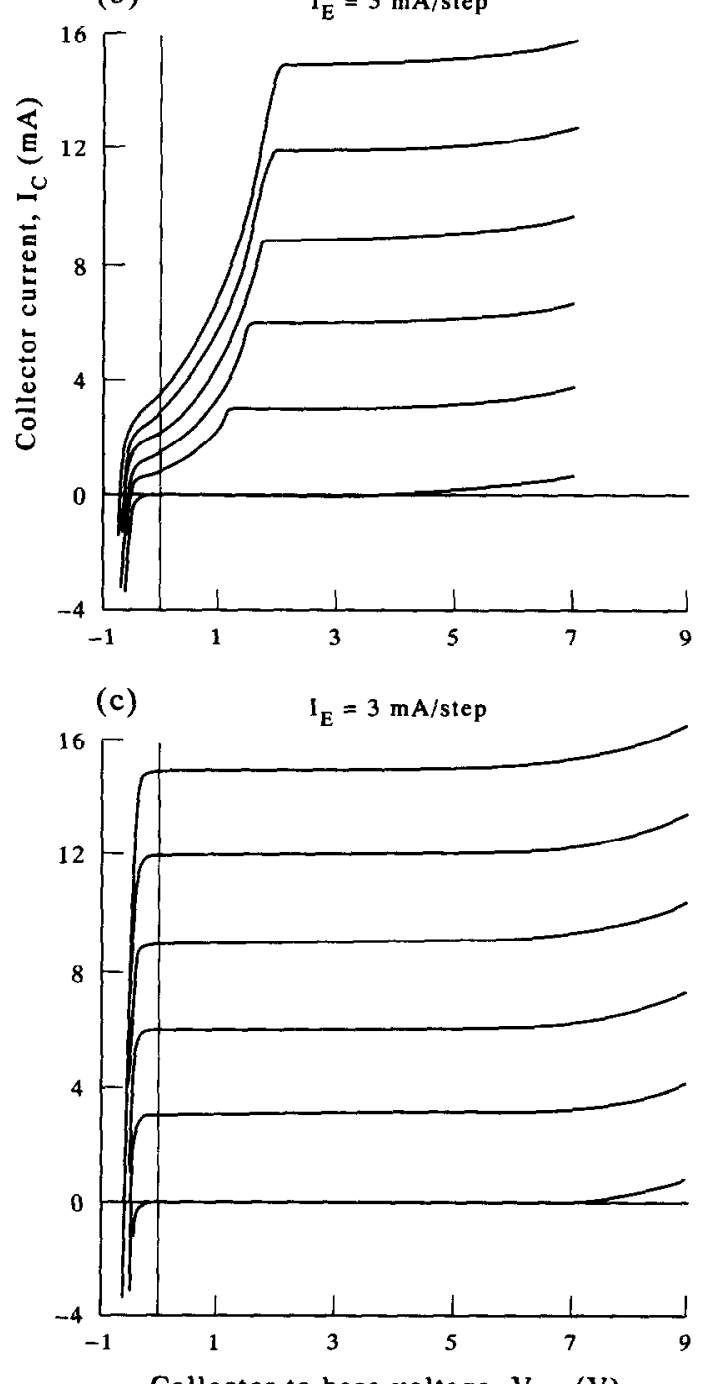

Collector to base voltage, $\mathrm{V}_{\mathrm{CB}}(\mathrm{V})$

Fig. 5. Common base output characteristics of DHBT's, (a) R423, (b) R424 and (c) R425. 


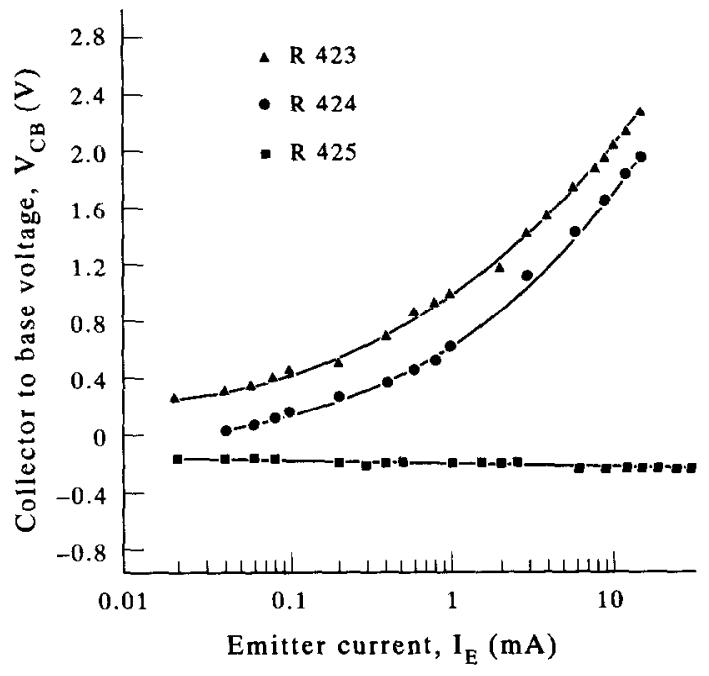

Fig. 6. Suppression voltage of DHBT's R423, R424 and R425 as functions of emitter current.

strongly collector current dependent. The larger the current, the larger the voltage. Again, these results confirm the previous discussion on the current dependent behavior of 2DEG density. The suppression voltage of R424 is slightly smaller than that of R423, because of the thicker undoped InGaAs spacer layer of R424. The suppression voltages of these two devices are around $2 \mathrm{~V}$ in magnitude, while the theoretical calculation based on the depletion approximation predicted that the voltage should be less than $0 \mathrm{~V}$ in both cases. This large difference suggests that the 2DEG in the heterojunction notch plays a very important role in the output characteristics of the InAlAs/InGaAs DHBT with abrupt basecollector junction. From Fig. 6, the "suppression voltage" of R425 is almost current independent because the heterojunction spike does not exist in its graded structure. The slight decline shown in the figure is the normal behavior of the emitter-base junction. When the emitter current increases, the emitter-base forward bias voltage has to increase, and an increase in base-collector forward bias voltage is needed to push the transistor into the saturation region. It follows that device $\mathbf{R} 425$ has a smaller suppression voltage in high emitter current region.

\section{CONCLUSIONS}

In conclusion, it is found that inserting an undoped InGaAs spacer layer between the base and collector of InAlAs/InGaAs DHBT can not eliminate the knee-shape and reach-through effects effectively. The base-collector heterojunction notch will accumulate a 2DEG and push up the heterojunction spike so as to cause these effects. A detailed mechanism has been presented. Although increased spacer-layer thickness may reduce these effects, the hot-electron-enhanced impact ionization limits the thickness to less than $500 \AA$-thick according to our experimental results. In the base-collector junction graded structure, the influences of the spike and the 2DEG have been removed. The results of our graded DHBT show no knee-shape and reach-through effects. The current gain and breakdown voltage are as high as 520 and $6 \mathrm{~V}$.

Acknowledgements-This study was supported by the National Science Council of the Republic of China under contract No. NSC-82-0404-E002-244 and the Telecommunication laboratory of the Republic of China under contract No. TL-NSC-82-5107.

\section{REFERENCES}

1. O. Sugiura, A. G. Dentai, C. H. Joyner, S. Chandrasekhar and J. C. Campbell, IEEE Electron Device Lett. 9, 253 (1988).

2. R. N. Nottenburg, J. C. Bischoff, M. B. Panish and H. Temkin, IEEE Electron Device Lett. 8, 282 (1987).

3. B. Jalali, R. N. Nottenburg, W. S. Hobson, Y. K. Chen, T. Fullowan, S. J. Pearton and A. S. Jordan, Electron. Lett. 25, 1496 (1989).

4. C. K. Peng, T. Won, C. W. Litton and H. Morkoc, IEEE Electron Device Lett. 9, 331 (1988).

5. L. Y. Leu, J. T. Gardner and S. R. Forrest, IEDM Tech. Dig., p. 149 (1990).

6. A. Dodabalapur and T. Y. Chang, IEEE Electron Device Lett. 12, 693 (1991).

7. H. H. Lin and C. H. Huang, Fourth Int. Conf. on IPRM, p. 381 (1992).

8. Y. K. Chen, R. N. Nottenburg, M. B. Panish, R. A. Hamm and D. A. Humphrey, IEEE Electron Device Lett. 10, 267 (1989).

9. M. S. Hybertsen, Appl. Phys. Lett. 58, 1759 (1991).

10. R. N. Nottenburg, Y. K. Chen, M. B. Panish, D. A. Humphrey and R. Hamm, IEEE Electron Device Lett. 10, 30 (1989).

11. H. Fukano, H. Nakajima, T. Ishibashi, Y. Takanashi and M. Fujimoto, IEEE Trans. Electron Devices 39, 500 (1992).

12. B. Jalali, Y. K. Chen, R. N. Nottenburg, D. Sivco, D. A. Humphrey and A. Y. Cho, IEEE Electron Device Lett. 11, 400 (1990).

13. C. Z. Chen and S. C. Lee, IEEE Trans, Electron Devices 34, 1463 (1987).

14. C. Z. Chen, S. C. Lee and H. H. Lin, J. appl. Phys. 62, 3976 (1987).

15. L. M. Su, N. Grote, R. Kaumanus and H. Schroeter, Appl. Phys. Lett. 47, 28 (1985).

16. T. Fujii, Y. Nakata, Y. Sugiyama and S. Hiyamizu, Jap. J. appl. Phys. 25, L254 (1986). 